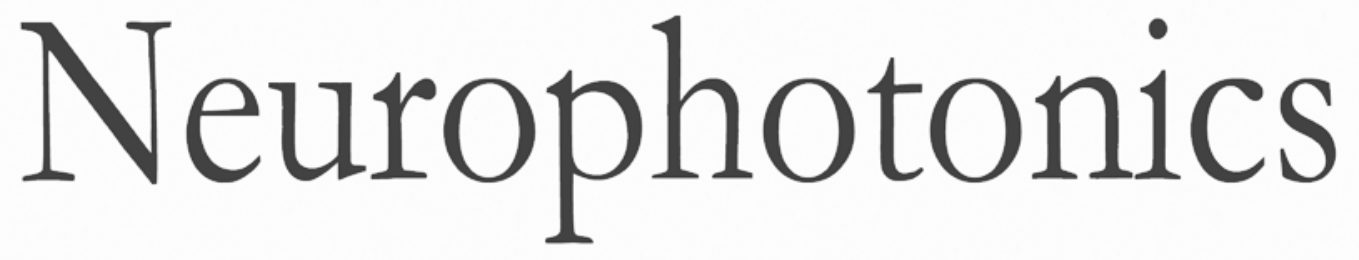

\title{
Pericyte structure and distribution in the cerebral cortex revealed by high- resolution imaging of transgenic mice
}

David A. Hartmann

Robert G. Underly

Roger I. Grant

Ashley N. Watson

Volkhard Lindner

Andy Y. Shih

\section{SPIE.}




\title{
Pericyte structure and distribution in the cerebral cortex revealed by high-resolution imaging of transgenic mice
}

\author{
David A. Hartmann, ${ }^{a}$ Robert G. Underly, ${ }^{a}$ Roger I. Grant, ${ }^{a, \dagger}$ Ashley N. Watson, ${ }^{a, \dagger}$ Volkhard Lindner, ${ }^{b}$ and \\ Andy Y. Shih ${ }^{\mathrm{a}, \mathrm{c}, \star}$ \\ a Medical University of South Carolina, Department of Neurosciences, 173 Ashley Avenue CRI 406, Charleston, South Carolina 29425, \\ United States \\ ${ }^{b}$ Maine Medical Center Research Institute, Center for Molecular Medicine, 81 Research Drive, Scarborough, Maine 04074, United States \\ 'Medical University of South Carolina, Center for Biomedical Imaging, 68 President Street, Charleston, South Carolina 29425, United States
}

\begin{abstract}
Pericytes are essential for normal brain function, but many aspects of their physiology remain enigmatic due to a lack of tools to genetically target this cell population. Here, we characterize brain pericytes using two existing Cre-recombinase driver mouse lines that can serve distinct purposes in cerebrovascular research. One line expresses an inducible version of Cre under the NG2 proteoglycan promoter, which provides the sparse labeling necessary to define the morphology of single cells. These mice reveal structural differences between pericytes adjacent to arterioles versus those broadly distributed in the capillary bed that may underlie differential roles in control of vessel caliber. A second line expresses Cre constitutively under the platelet-derived growth factor receptor $\beta$ promoter and provides continuous, highly specific and near-complete labeling of pericytes and myocytes along the entire cerebrovasculature. This line provides a three-dimensional view of pericyte distribution along the cortical angioarchitecture following optical clearing of brain tissue. In combination with recent reporter lines for expression of optogenetic actuators and activity-sensitive probes, these mice may be key tools for studying pericyte biology in the intact brain. () 2015 Society of Photo-Optical Instrumentation Engineers (SPIE) [DOI: 10.1117/1.NPh.2.4.041402]
\end{abstract}

Keywords: microcirculation; capillary; cerebral blood blow; two-photon microscopy; pericyte; smooth muscle.

Paper 15009SSR received Feb. 2, 2015; accepted for publication Mar. 27, 2015; published online May 27, 2015.

\section{Introduction}

Pericytes ${ }^{1}$ play diverse roles in the brain including maintenance of blood-brain barrier integrity, ${ }^{2,3}$ angiogenesis, ${ }^{4}$ regulation of cerebral blood flow, ${ }^{5,6}$ clearance of cellular debris, ${ }^{7}$ and generation of pluripotent cells. ${ }^{8}$ Defects in pericyte function also facilitate tissue injury in stroke ${ }^{5}$ and neurodegeneration by impairing cerebral microcirculation ${ }^{9}$ and vascular stability. ${ }^{6,10}$ This provides a strong impetus to understand the beneficial and deleterious roles of pericytes in health and disease.

Much of our understanding of these enigmatic cells come from ex vivo studies using histology, electron microscopy, or slice physiology, where pericytes can be identified by morphology or staining with a battery of protein markers. ${ }^{11}$ However, the translation of this work to an in vivo preparation has been hampered by a lack of tools to selectively target pericytes with transgene expression. In particular, methods to specifically and chronically express bright fluorescent proteins in pericytes would aid their identification during fluorescence imaging studies. Further, targeted expression of genetically encoded tools to interrogate $^{12}$ or modulate ${ }^{13}$ cell activity can provide significant advances in our understanding of pericyte physiology. Thus far, in vivo fluorescence imaging of pericytes has been restricted to two transgenic mouse lines: NG2-DsRed ${ }^{5}$ and $\beta$-actin-GFP mouse. ${ }^{14}$ Both lines are sufficient to report the location of

\footnotetext{
*Address all correspondence to: Andy Y. Shih, E-mail: shiha@musc.edu
}

†These authors contributed equally. pericytes based on their stereotyped fusiform or ovoid cell bodies that protrude from the vasculature. However, these animals do not support future goals to genetically target cerebral pericytes with transgenes for interactive studies of their physiology. Moreover, neither line enables visualization of the farreaching processes, which constitute the majority of the vascular coverage offered by pericytes and are likely essential for bloodbrain barrier stability.

Cre-lox recombination is widely used to carry out genetic manipulations in mouse ${ }^{15}$ including the introduction of ectopic genes by breeding Cre-recombinase driver lines with ubiquitously floxed reporters. ${ }^{16,17}$ The cellular specificity of expression is achieved primarily through the promoter that gates Crerecombinase expression. Here, we evaluate two Cre lines, one driven by the promoter for neural/glial antigen 2 (NG2) proteoglycan ${ }^{18}$ and the other by platelet-derived growth factor receptor $\beta$ (PDGFR $\beta$ ), ${ }^{19}$ to determine the specificity and extent of pericyte targeting. Both proteins are widely accepted immunohistochemical markers for pericytes. ${ }^{11}$ However, they are also expressed by other brain cells, and thus require characterization of gene targeting specificity. For example, in past work, we found that the constitutive expression of Cre by the NG2 promoter led to widespread targeting of neurons, in addition to oligodendrocyte precursors and pericytes, rendering these animals unusable for in vivo imaging due to the sheer density of labeling. ${ }^{17}$ Thus, it was necessary to characterize inducible Cre

2329-423X/2015/\$25.00 (C) 2015 SPIE 
animals and also to identify new lines with greater targeting specificity.

Our results show that the NG2-CreER ${ }^{\mathrm{TM}}{ }^{20}$ and PDGFR $\beta$ $\mathrm{Cre}^{19}$ lines possess complementary attributes for studies of pericyte structure and function. The combined use of these animals will facilitate in vivo studies from the level of single pericytes to populations of pericytes comprising tens to hundreds of overlapping cells. Sparse labeling provided by the NG2-CreER line presents the first clear view of the structure of single pericytes, while broad homogeneous labeling with the PDGFR $\beta$-Cre line provides key information about the distribution and topological localization of pericytes in the cerebral microvasculature.

\section{Results}

Male heterozygous NG2-CreER or PDGFR $\beta$-Cre driver mice were bred with female Ai14 reporter mice ${ }^{16}$ created by the Allen Institute to induce expression of the bright red fluorescent protein, tdTomato. ${ }^{21}$ The progeny of these crosses were termed NG2-tdTomato and PDGFR $\beta$-tdTomato mice, respectively.
The NG2-tdTomato line was inducible and required a period of tamoxifen injections during adulthood to initiate tdTomato expression, whereas the PDGFR $\beta$-tdTomato line expressed tdTomato constitutively. We focused primarily on transgene expression in the cerebral cortex where in vivo two-photon imaging is commonly performed, ${ }^{22}$ although other regions of the central nervous system (CNS) were also examined.

\subsection{Pericyte Labeling in the Central Nervous System of NG2-tdTomato and PDGFR $\beta$ - tdTomato Mice}

We first direct our attention to the NG2-tdTomato line [Figs. 1(a)1(c)]. This mouse line targets pericytes, but also oligodendrocyte precursors and neurons. However, tdTomato fluorescence appeared brightest in pericytes allowing for straightforward identification in histology based on morphology and perivascular localization (ovoid cell body with processes running along the microvasculature) [Fig. 1(c)]. Pericyte labeling was found to be incomplete with a common regimen of tamoxifen injections

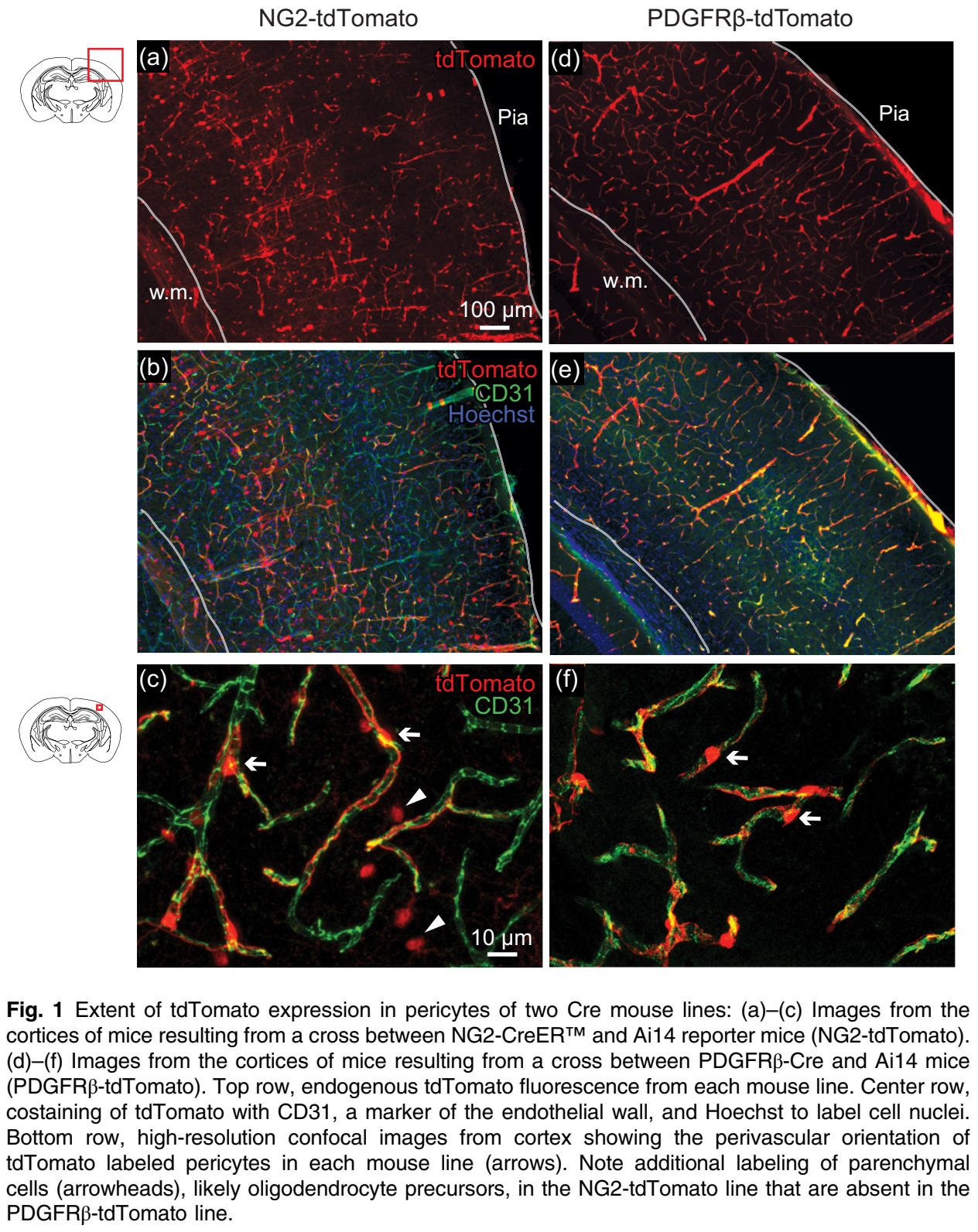


(100 mg/kg tamoxifen i.p. per day for 5 days). ${ }^{16}$ The distribution of labeled pericytes was heterogeneous, with localized regions that exhibited near-complete pericyte labeling, referred to as hotspots, and other areas nearly devoid of labeling. In contrast to the heterogeneity observed in the NG2-tdTomato line, pericyte labeling was contiguous in the capillary beds of PDGFR $\beta$-tdTomato mice [Figs. 1(d)-1(f)]. Pericyte processes were found to cover the entire microvasculature such that the vascular topology could be distinguished without the need for a separate vascular label. While the cell bodies of pericytes could be easily detected, the shape of a single cell was no longer discernible as there was no obvious separation between processes of neighboring cells [Fig. 1(f)].

To further characterize the extent and specificity of pericyte labeling, we tested a number of antibodies specific for established pericyte markers, namely CD13 (aminopeptidase N), ${ }^{23}$ PDGFR $\beta,{ }^{24}$ and $\mathrm{NG}^{18}$ (Fig. 2). While all antibodies were capable of labeling pericytes, in our hands only CD13 staining provided the specificity that was necessary for quantitative analysis of pericyte number [Figs. 2(a) and 3(a)]. This was because the NG2 and PDGFR $\beta$ antibodies we used labeled other cell types with higher or equivalent density such that the pericytes could not be distinguished from observing the stain alone [Figs. 2(b) and 2(c)]. Using CD13 as a normalization factor for all pericytes within the tissue section, we found that NG2-tdTomato mice labeled $235 \%$ of CD13-positive pericytes in cerebral cortex (Table 1, sensitivity). However, the variance was high, with sensitivity ranging between $0 \%$ and $86 \%$, when small regions of interest were examined. In areas of sparse labeling, the complete morphology of individual pericytes could be visualized [Figs. 1(a) and 2].

Immunohistochemical analysis of PDGFR $\beta$-tdTomato mice revealed that $\sim 99 \%$ of CD13-positive pericytes were labeled (Table 1, sensitivity) and that labeling was homogeneous throughout the cortex [Figs. 1(d) and 3]. The specificity of tdTomato expression for pericytes was remarkably high, as only a rare neuron was found labeled within the parenchyma. Notably, since CD13 appeared to colabel the vast majority of pericytes in this line, we suggest that CD13 is an all-encompassing detector for pericytes in mouse cortex. ${ }^{25}$ Further, since the PDGFR $\beta$ tdTomato line exhibited almost no targeting to parenchymal cells, the false-positive rate for mistaking a nonpericyte tdTomato-positive cell for a pericyte was negligible compared with the NG2-tdTomato line (Table 1, false positive rate).

Finally, labeling of pericytes in other CNS areas, including hippocampus, striatum, olfactory bulb, cerebellum, and spinal
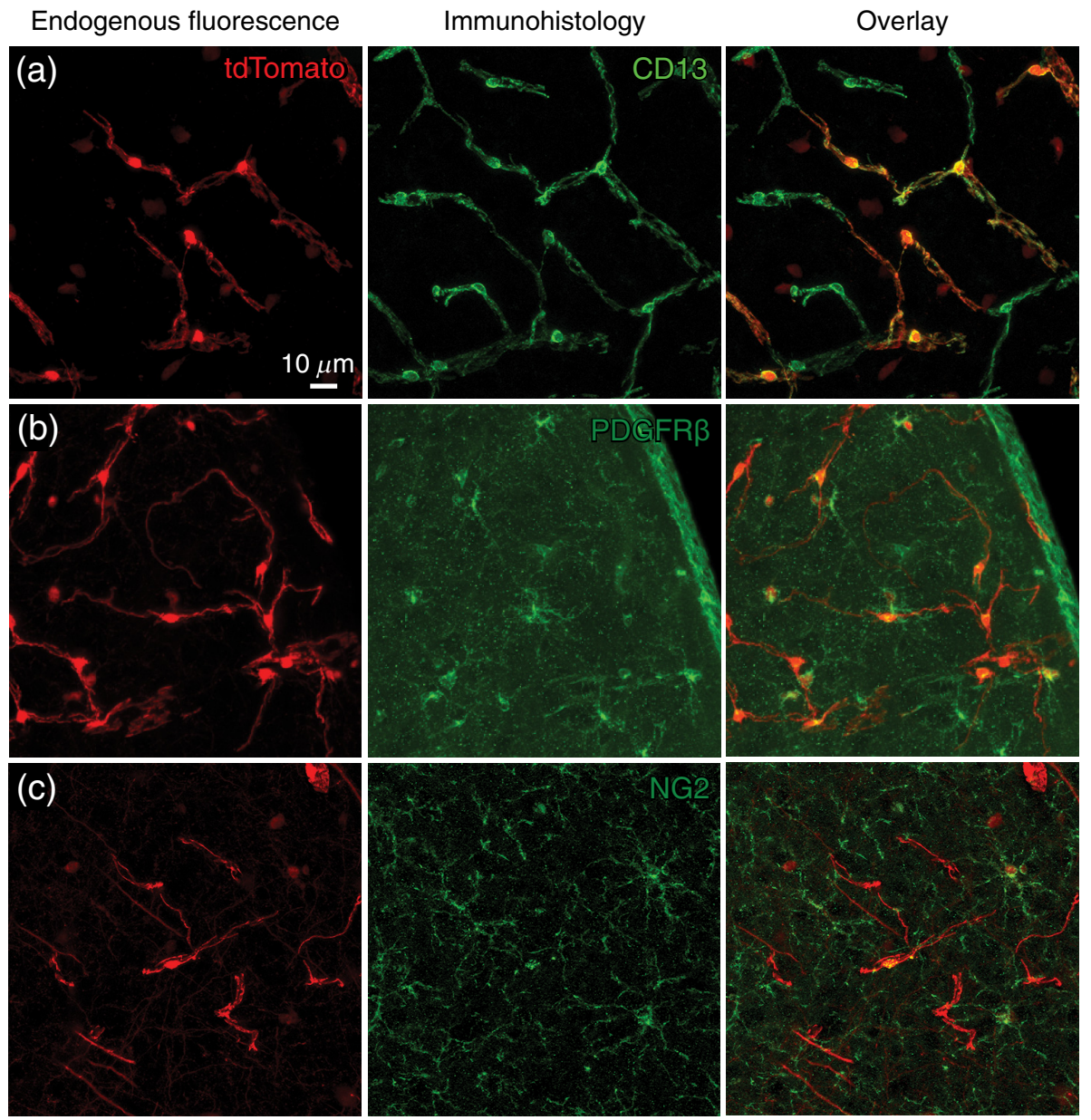

Fig. 2 Validation of pericyte targeting in NG2-tdTomato mice. Left column shows the endogenous tdTomato fluorescence from regions in the sensory cortex. The center column shows staining with verified immunohistochemical pericyte markers. Three established pericyte markers, (a) CD13, (b) PDGFR $\beta$, and (c) NG2 were examined. The right column shows an overlay of endogenous and immunostained fluorescence. All images are maximal projections of image stacks collected by high-resolution confocal imaging. 

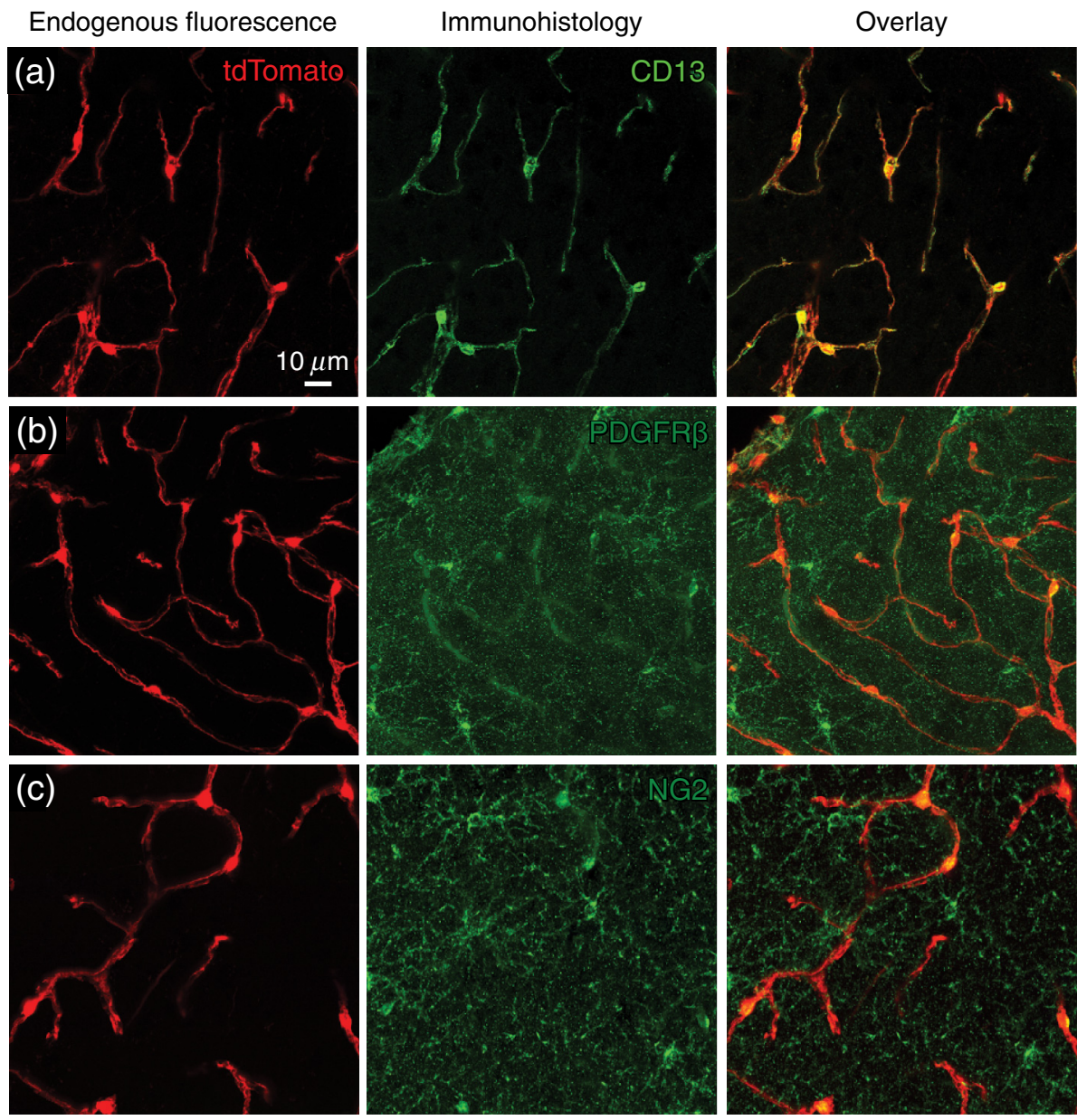

Fig. 3 Validation of pericyte targeting in PDGFR $\beta$-tdTomato mice. Left column shows the endogenous tdTomato fluorescence from regions in the sensory cortex. The center column shows the staining with verified immunohistochemical pericyte markers. Three established pericyte markers, (a) CD13, (b) PDGFR $\beta$, and (c) NG2 were examined. The right column shows an overlay of endogenous and stained fluorescence.

cord, was similar to that described for the cortex (Fig. 4). Both lines may also be useful for visualizing pericytes in organs other than the brain, although this was not examined in the current study.

\subsection{In Vivo Imaging of Pericytes in the Sensory Cortex}

Following validation of tdTomato expression in pericytes, we examined whether both mouse lines were suitable for visualizing pericytes using in vivo two-photon imaging. ${ }^{26} \mathrm{We}$

Table 1 Quantification of pericyte targeting in NG2-tdTomato and PDGFR $\beta$-tdTomato lines.

\begin{tabular}{lccc} 
& $\begin{array}{c}\text { Number of } \mathrm{CD}^{+} 3^{+} \\
\text {pericytes per mm² } \\
\text { (mean } \pm \text { s.d.) }\end{array}$ & $\begin{array}{c}\text { Sensitivity } \\
(\%)\end{array}$ & $\begin{array}{c}\text { False } \\
\text { positive } \\
\text { rate (\%) }\end{array}$ \\
\hline $\begin{array}{l}\text { Genotype } \\
\text { NG2-tdTomato (1827 } \\
\text { pericytes from six mice) }\end{array}$ & $227.9 \pm 30.7$ & $35.6 \pm 8.4$ & $4.3 \pm 2.4$ \\
$\begin{array}{l}\text { PDGFR } \beta \text {-tdTomato (952 } \\
\text { pericytes from three mice) }\end{array}$ & $237.5 \pm 12.4$ & $99.4 \pm 0.7$ & $0.3 \pm 0.3$ \\
\hline
\end{tabular}

created skull-removed cranial windows and imaged upper cortical layers using a 975-nm excitation. ${ }^{27}$ As expected, tdTomatopositive pericytes fluoresced brightly from the cell body down to distal processes in both lines (Fig. 5). In the heterogeneously labeled NG2-tdTomato line, it was possible to find imaging fields rich with pericytes (Fig. 5, top row) directly adjacent to fields with sparse labeling (not shown) all within a single cranial window. As expected from histological examination, the PDGFR $\beta$-tdTomato line showed contiguous labeling of pericytes in all cortical areas examined (Fig. 5, bottom row).

\subsection{Morphological Characteristics of Individual Cortical Pericytes}

The true structure of a pericyte has remained elusive because immunohistochemical labeling either reports subcellular structures or leads to contiguous staining that does not allow adjacent pericytes to be visually separated. Regions of sparse pericyte labeling in the NG2-tdTomato line provided a unique opportunity to define the morphology of individual pericytes including fine distal branches. Using confocal microscopy, we collected high-resolution volumetric data from isolated, single cells. Pericytes as a population were highly variable in structure (Fig. 6). The processes extending from the cell body, however, were 


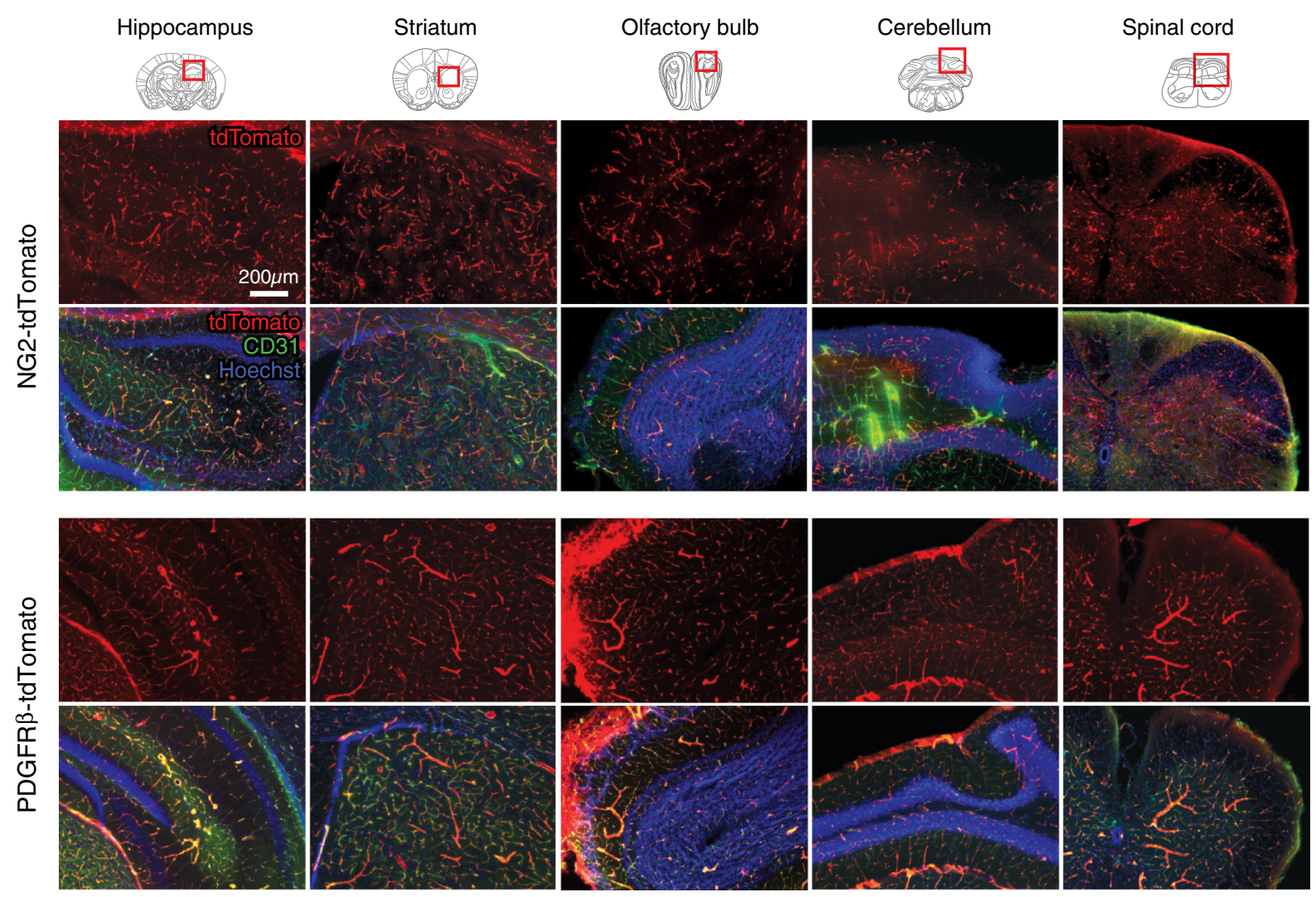

Fig. 4 Transgene expression in different brain regions of adult NG2-tdTomato and PDGFR $\beta$-tdTomato mice. Epifluorescence images for each animal show endogenous tdTomato fluorescence (top panel) and are then overlaid with CD31 and Hoechst labeling (bottom panel).
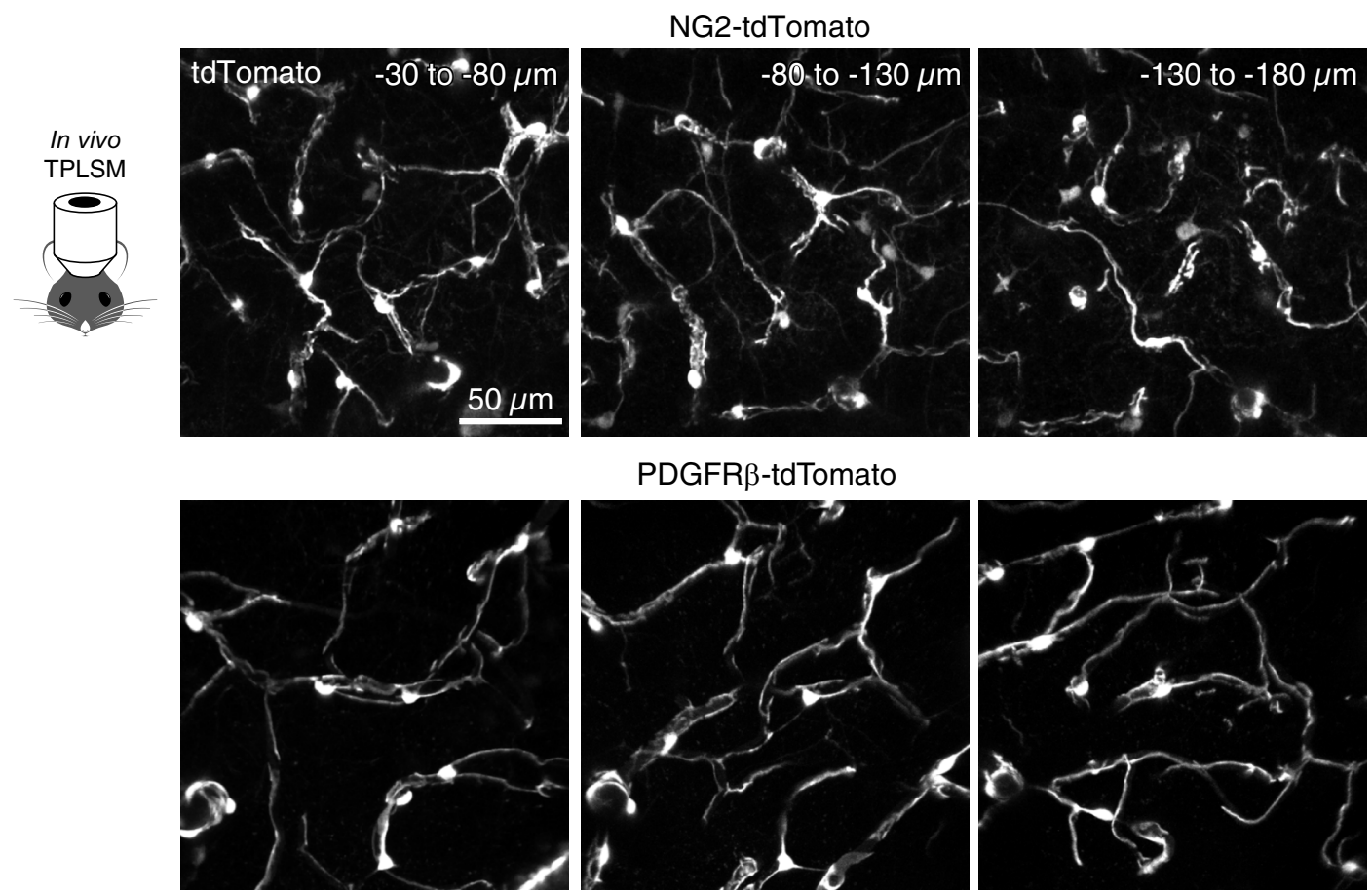

Fig. 5 Visualization of pericytes in vivo with two-photon laser-scanning microscopy. Pericytes imaged in the upper cortical layers of NG2-tdTomato (top row) or PDGFR $\beta$-tdTomato (bottom row) mice. Images are maximally projected stacks spanning $50 \mu \mathrm{m}$ of tissue thickness at various depths below the pial surface. A hotspot enriched with pericytes was imaged in the NG2-tdTomato mouse. 
sufficiently stereotyped to allow for categorization. To organize pericyte branching topology, we denoted processes as a "primary trunk" if they protruded directly from the cell body, and as "primary branches" if they split from the primary trunk to follow another trajectory at a vascular junction [Fig. 6(a)]. Primary processes were never found to branch beyond more than one capillary junction. Short projections occasionally emerged from the primary processes, extending roughly perpendicularly to wrap the microvessel lumen [Fig. 6(b)]. These were referred to as secondary processes for consistency with past work. $^{28}$

In its simplest form, primary trunks and branches were thin singular strands of approximately $2 \mu \mathrm{m}$ in diameter [Figs. 6(b) and 6(f)]. The secondary processes emerging from these thin strands were easily distinguished. Primary processes also existed as pairs that coursed along a microvessel on opposing sides of the lumen. These pairs often appeared twisted, forming helical structures that were interweaved by fine connections [Figs. 6(d) and 6(e)]. In its most complex form, primary trunks formed a mesh-like sheath that surrounded the entire vessel [Figs. 6(b), 6(c), and 6(g)]; these pericytes will be referred to as "mesh pericytes." Mesh pericytes were more common to microvessels of larger diameter, i.e., 6 to $10 \mu \mathrm{m}$ in diameter. In the case of both helical and mesh processes, their extensive interconnections may have been elaborations of secondary processes that existed as single protrusions on single strand primary processes. An additional feature of pericytes was the location of their cell bodies along the microvasculature. The cell bodies could be located at the bifurcation of vessels, i.e., junctional, or along the capillary segment between junctions, i.e., en passant [Figs. 6(d) and 6(e)].

We further characterized pericyte processes by measuring their numbers and lengths. We randomly sampled the cortices of NG2-tdTomato mice for isolated pericytes. Since capillary beds constitute the vast majority of the subsurface microvasculature, this cell population was comprised primarily of pericytes extending thin strand or helical processes that resided on microvessels of 4 to $6 \mu \mathrm{m}$ in diameter. These pericytes extended between two and four primary trunks, with a median of 3 ( $n=14$ cells) [Fig. 6(h)]. Individual primary trunks would then split once, at most, to generate two primary branches. The total number of branches per cell, however, was highly variable ranging from 0 to 7 with a median of 4 . Primary branches were also shorter than primary trunks, averaging $41 \pm 31$ and $67 \pm 41 \mu \mathrm{m}$ in length, respectively (mean \pm s.d., $p=0.003$; Kolmogorov-Smirnov test) [Fig. 6(i)]. Given these parameters, we estimate that a single pericyte within the capillary bed covers $\sim 300 \mu \mathrm{m}$ of total capillary length.

Past work has suggested that the pericytes more closely associated with penetrating arterioles are regulators of blood flow. ${ }^{5}$ It is thus conceivable that such pericytes are distinct in structure compared with their counterparts deeper in the capillary beds. To identify these pericytes, we immunohistochemically stained for $\alpha$-smooth muscle actin ( $\alpha$-SMA). While we were unable to detect strong $\alpha$-SMA expression in pericytes as reported in some peripheral vascular beds, ${ }^{29}$ labeling of parenchymal arterioles was very robust. In these tissues, we could follow the hierarchy of arteriole branches as they ramified from penetrating arterioles to precapillary arterioles to capillaries and the mural cell types covering each topological level [Figs. 7(a)-7(g)]. Here, we specifically define precapillary arterioles as subsurface branches of the penetrating arterioles immediately preceding the $\alpha$-SMA
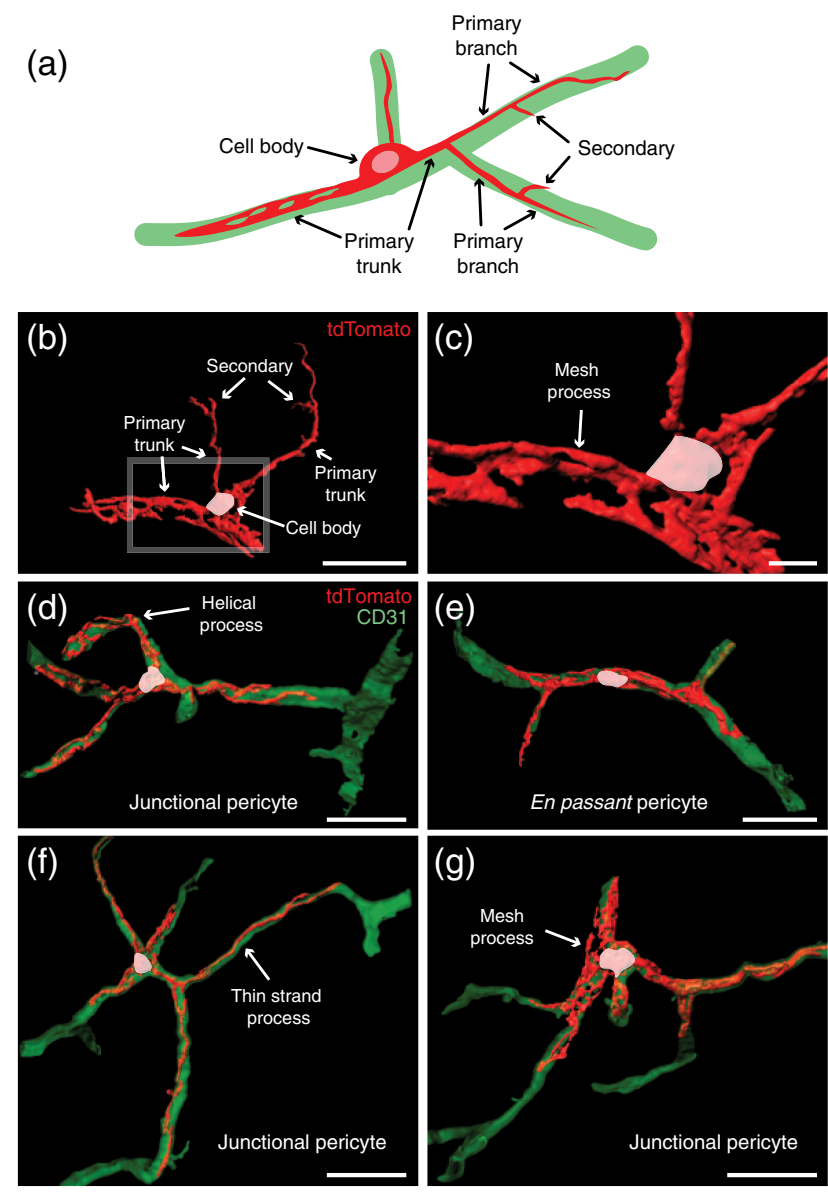

(h) - $\quad$ D Primary trunk

(i)
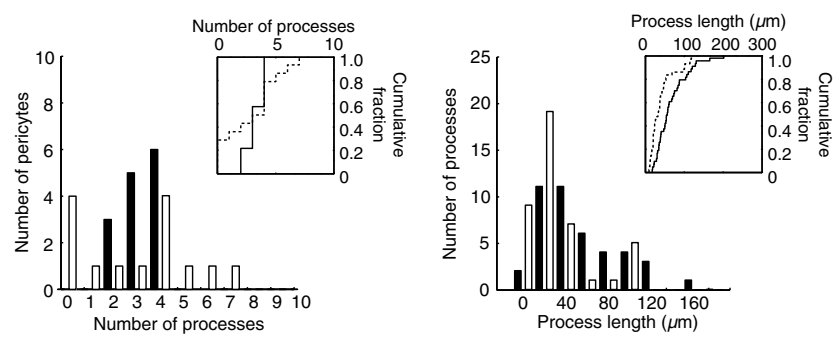

Fig. 6 Structural characteristics of individual pericytes revealed by NG2-tdTomato mice: (a) Schematic of a junctional pericyte with classification of perivascular processes. (b) Three-dimensional surface rendering of a pericyte with three primary trunk processes, which extend directly from the cell body. Small secondary processes can be seen extending perpendicularly from the primary process. The cell body is shown in white. (c) Magnified view of a mesh process that ensheaths the microvascular lumen [inset from (b)]. (d) Example of a junctional pericyte with helical primary trunk processes. (e) Example of an en passant pericyte. (f) A complex pericyte with four primary trunks, one of which splits into two primary branches. The processes of this pericyte are thin singular strands. (g) Example of a mesh pericyte that likely ensheaths a postcapillary venule. (h) Histogram and cumulative fraction plot of the number of each process type, primary trunk or branch, possessed by individual pericytes. (i) Histogram and cumulative fraction plot of the length of each process type. Scale bar for all images is $50 \mu \mathrm{m}$, with the exception of (c), which is $10 \mu \mathrm{m}$.

lacking capillary bed. This transition point was referred to as an arteriole-capillary interface [Figs. 7(a), 7(h), and 7(i)]. We identified isolated tdTomato-positive pericytes that were directly juxtaposed to $\alpha$-SMA-rich cells at this interface [Figs. 7(j) and $7(\mathrm{k})$ ]. These pericytes expressed very low levels of $\alpha$-SMA 
comparatively [Fig. 7(i)], always exhibited mesh processes, and appeared to tightly interlock with a smooth muscle-pericyte hybrid cell type (see below) on the arteriolar side of the interface. The primary trunks of these mesh pericytes $(n=7$ cells examined) were typically fewer in number (median of 2 versus $3)$ and shorter in length $(40 \pm 35 \mu \mathrm{m}$ versus $70 \pm 40 \mu \mathrm{m}, p=$ 0.001 , mean \pm s.d.; K-S test) compared with the pericytes of the capillaries [Figs. 6(h) and 6(i)]. The primary trunks also exhibited a slightly higher surface to volume ratio $(1.8 \pm 0.3$ versus $1.6 \pm 0.4, p=0.02$, mean \pm s.d.; $\mathrm{K}-\mathrm{S}$ test), suggesting a greater structural complexity that may be important for contractile function. In contrast, we found no significant differences in the morphology of primary branches between mesh pericytes and capillary pericytes. Finally, we also observed mesh pericytes enwrapping larger diameter subsurface microvessels that were not associated with $\alpha$-SMA that were likely
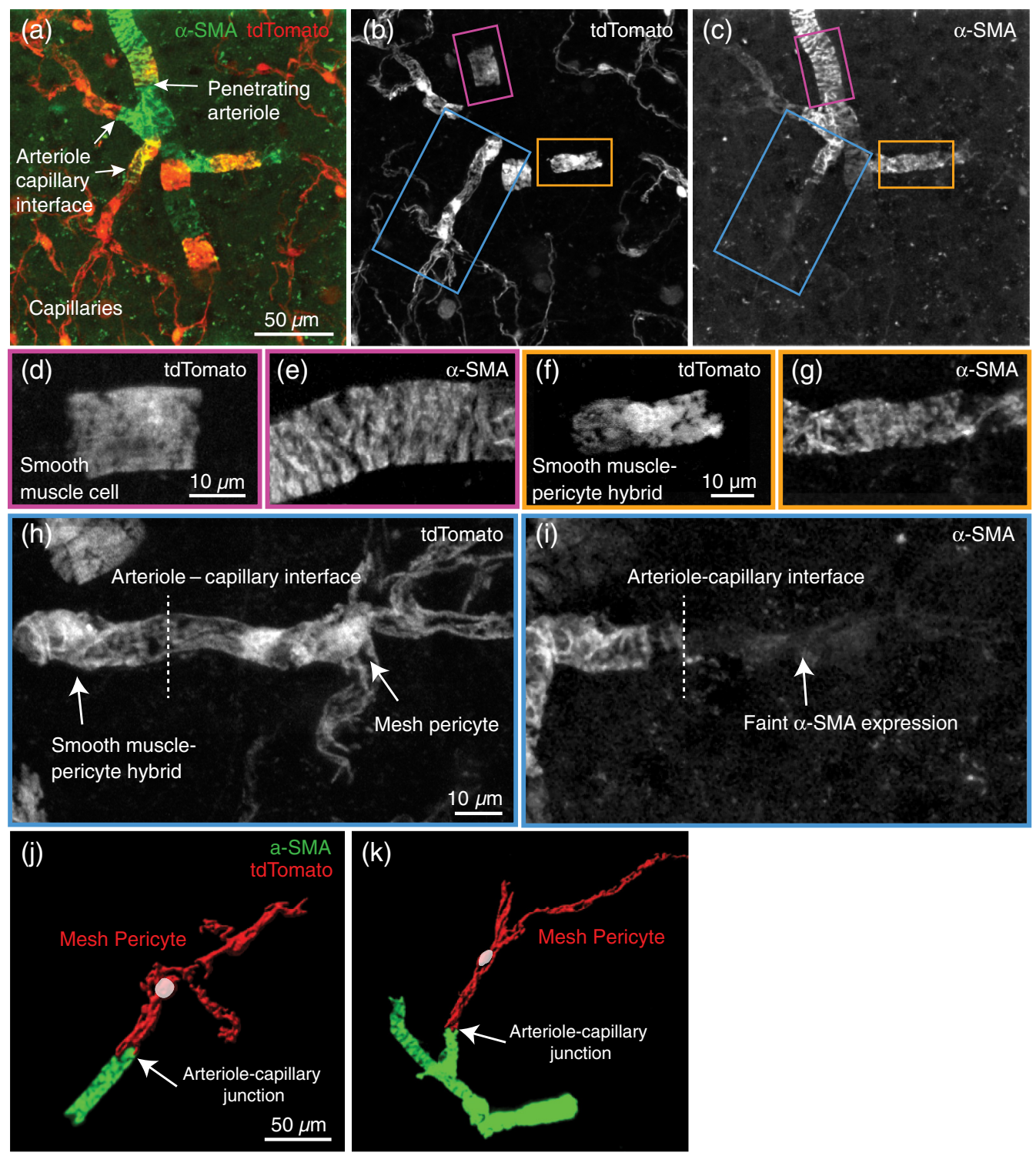

Fig. 7 Mural cells of the arteriole-capillary interface: (a) Branching of a penetrating arteriole gives rise to subsurface precapillary arterioles and capillaries. Immunostaining reveals the point of transition between $\alpha$-SMA-rich arterioles and $\alpha$-SMA-poor capillaries. This transition point is termed the arteriole-capillary interface. The image shown is a maximally projected confocal image stack taken from the cortex of an NG2-tdTomato animal. Separation of the (b) red tdTomato and (c) green $\alpha$-SMA channels reveals the morphology of individual mural cells along this vascular hierarchy. (d, e) A smooth muscle cell of the penetrating arteriole that expresses $\alpha$-SMA. (f, g) A smooth muscle-pericyte hybrid located on the first branch of the penetrating arteriole. Note the substantial expression of $\alpha$-SMA and brightly labeled ovoid cell body, but lack of classic pericyte phenotype. (h, i) Magnified view of an arteriole-capillary interface. A tdTomato-expressing smooth muscle-pericyte hybrid and mesh pericyte are adjacent to each other and thus not visually separable. However, $\alpha$-SMA labeling ceases at the interface between the two cells. The mesh pericyte downstream of the interface exhibits comparatively minute levels of $\alpha$-SMA. (j, k) Two examples of isolated tdTomato-positive mesh pericytes at the arteriole-capillary interface. These mesh pericytes interlock, rather than overlap, with smooth muscle-pericyte hybrids. The pericyte cell body is shown in white. 
postcapillary venules. Collectively, these data support the idea that the cerebral pericytes are a diverse group of cells that vary in morphology along the cerebral vascular topology and likely serve different functions in blood flow control and blood-brain barrier maintenance.

\subsection{Continuum of Cerebrovascular Mural Cells}

In addition to the labeling of pericytes, NG2-tdTomato mice also revealed expression in other isolated mural cells. All pial arteries and arterioles exhibited intermittent labeling of smooth muscle cells that appeared as concentric rings surrounding the lumen [Fig. 8(a)]. Smooth muscle cells maintained their ring shape well into the parenchyma along penetrating arterioles. As penetrating arterioles ramified into smaller precapillary arterioles, the mural cells exhibited a mixed phenotype of smooth muscle and pericyte [Fig. 8(b)]. These cells, termed "smooth musclepericyte hybrids" were slightly more elongated than smooth muscle cells, enveloped the entire endothelium, and possessed protruding, ovoid cell bodies similar to those of pericytes. They also expressed levels of $\alpha$-SMA comparable with larger upstream arterioles [c.f. Figs. 7(d) and 7(e) to 7(h) and 7(i)]. These hybrid cells appear to make direct contact with the mesh pericytes at the arteriole-capillary interface and were observed at all interfaces examined [Figs. 7(h) and 7(i)]. On collecting venules, we observed stellate-shaped cells that ensheathed the vessel lumen with broad "leaf-like" processes, as previously described in ultrastructural studies ${ }^{28}$ [Fig. 8(c)]. These cells have been categorized as smooth muscle cells of venules by past work. ${ }^{11}$ The similarities between our results and those of previous ultrastructural electron microscopy studies $^{28}$ suggest that tdTomato is present throughout the pericyte cytoplasm. In the PDGFR $\beta$-tdTomato line, we observed the complete labeling of mural cells from the pial surface down to the capillary beds [Figs. 8(d) and 8(e)]. Similarly, labeling of cells along venules was also contiguous [Fig. 8(f)].

\subsection{Wide-Field Imaging of Pericyte Distribution in Optically Cleared Tissues}

Large-scale characterizations of pericyte distribution may improve our understanding of blood flow regulation or
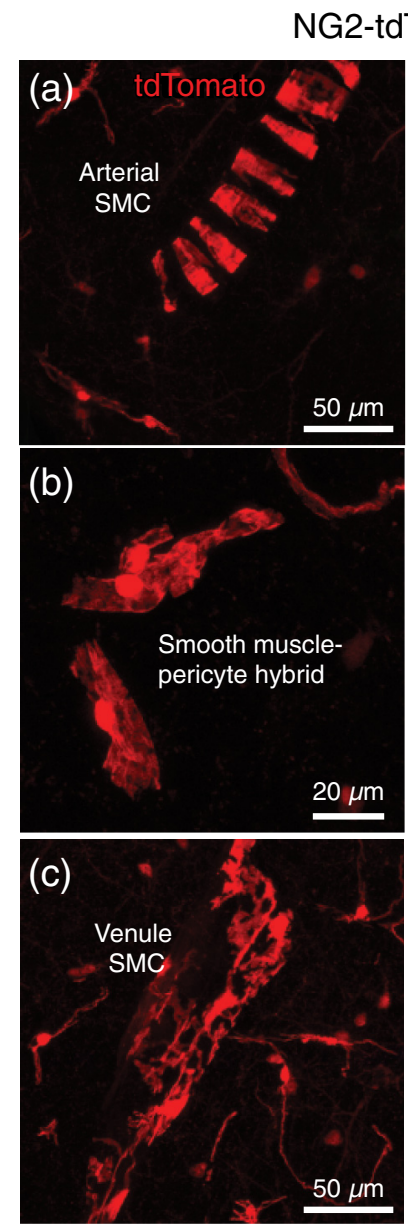
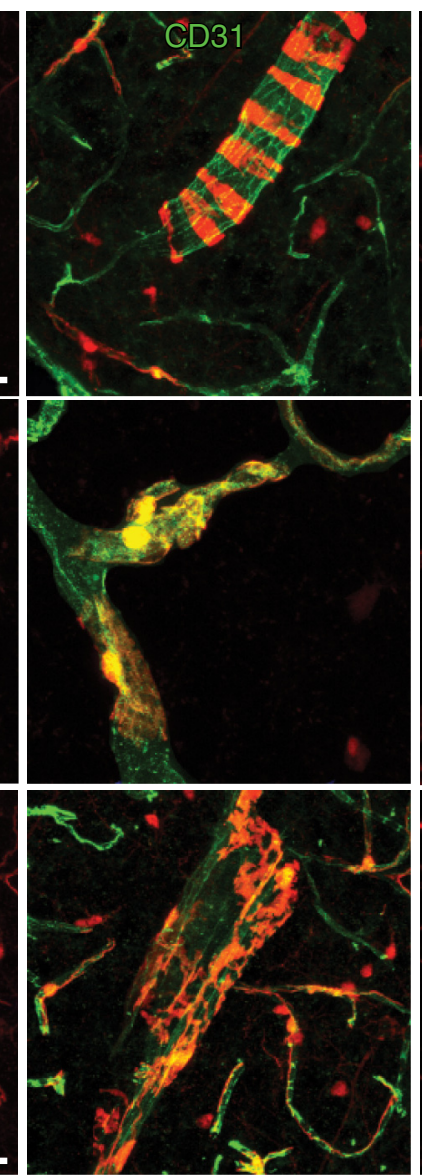

PDGFR $\beta$-tdTomato
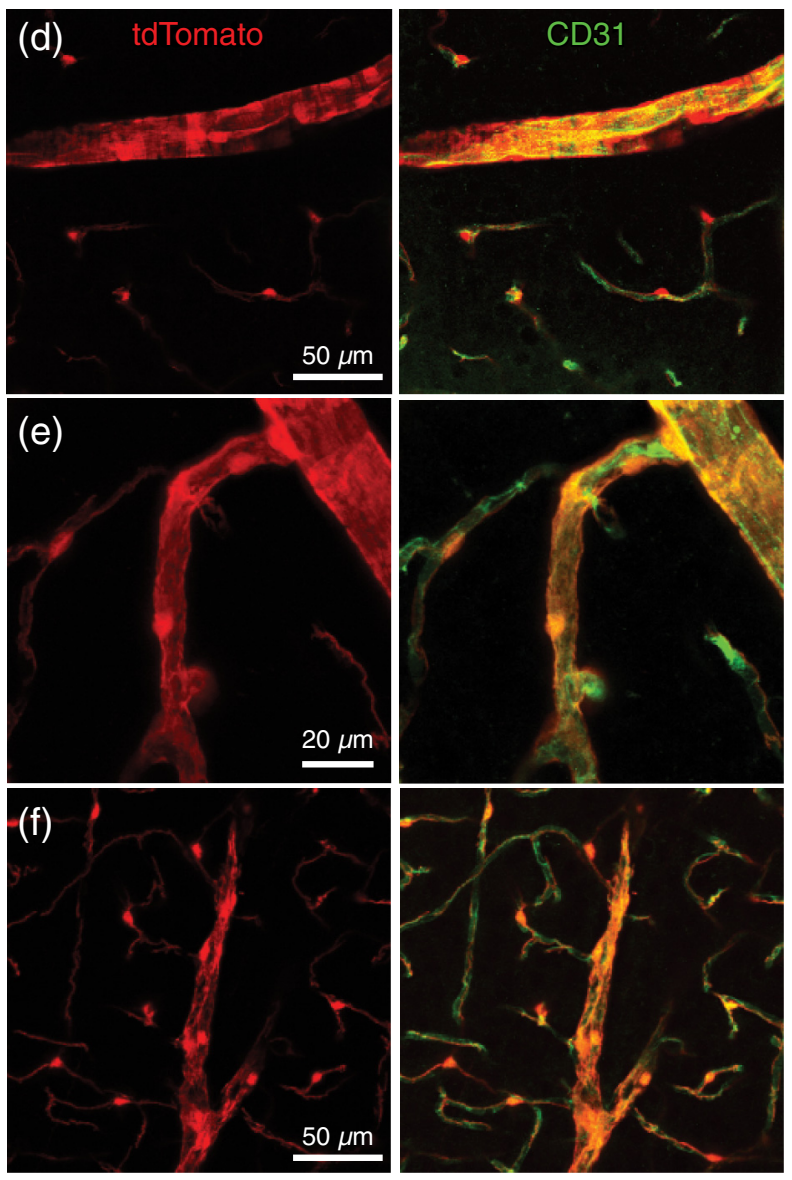

Fig. 8 Arteriolar and venular mural cells labeled in NG2-tdTomato and PDGFR $\beta$-tdTomato mice: (a) High-resolution confocal image projections of ring-shaped smooth muscle cells (SMC) in pial arterioles of NG2-tdTomato mice. Note that incomplete labeling allows individual cells to be discerned. (b) Hybrid smooth muscle-pericyte cells residing on precapillary arterioles exhibit ovoid cell bodies but lack the long-reaching processes common to capillary pericytes. (c) Stellate-shaped smooth muscle cells cover the walls of a collecting venule. Note the flat "leaf-shaped" processes that spread over the vessel surface. (d)-(f) Equivalent vascular structures imaged in the PDGFR $\beta$-tdTomato line are contiguous throughout the cerebrovasculature. Thus, individual cell shapes cannot be readily discerned. 
differential vascular vulnerabilities in disease. The near-complete labeling of pericytes in the PDGFR $\beta$-tdTomato line provided a means to obtain these metrics when combined with optical tissue clearing and high-resolution imaging. tdTomato fluorescence was well preserved in cleared tissues, providing image stacks with a strong signal over the entire depth of the cortex [Fig. 9(a)].

As an initial realization of this approach, we examined three datasets to test the hypothesis that pericyte numbers covary with increased neural density, perhaps as a means to more precisely modulate blood flow for local metabolic demand. We focused on cortical layers I and II/III, which are readily accessible by in vivo imaging [Figs. 9(b)-9(d)]. Layer II/III exhibited an approximately fourfold higher cell density compared with layer I, mostly attributable to an increase in neurons [Figs. 9(d) and $9(\mathrm{~g})] .{ }^{30}$ Interestingly, in contrast to our postulation, we found that the pericyte numbers decreased by $40 \%$ in layer II/III [Figs. 9(b) and 9(e)]. This was not due to a commensurate decrease in vascular volume, as only a modest reduction of $7 \%$ was observed in layer II/III compared with layer I [Figs. 9(c) and 9(f)]. This suggests that the pericyte density is not static through different cortical layers, and their distribution may impact flow in a layer specific manner.

Pericytes located at capillary junctions may be capable of regulating red blood cell passage through two or more routes of flow and may thus be more potent modulators than their en passant counterparts. Of all the pericytes examined $(n=105)$, $56 \%$ of the pericytes had cell bodies located at a capillary junction, while $38 \%$ were en passant. Many pericytes appeared to be at a junction, but exhibited cell bodies that were at least a cell width away from the junction upon closer inspection. The remaining $6 \%$ were undefined due to their location at the edge of the imaged tissue volume. These relative percentages did not change significantly between layers I and II/III. In addition, only $28 \%$ of all capillary junctions examined $(n=217)$ were truly ensheathed by the cell body of a pericyte.

\section{Discussion}

We have characterized two Cre-recombinase mouse lines that enable genetic targeting of cerebrovascular pericytes. We show that an inducible line, driven by expression through the NG2 promoter, ${ }^{20}$ provides regions of sparse labeling that enabled us to define pericyte structures that could not be otherwise resolved with immunostaining. These data show that the pericyte processes are highly varied, with shapes ranging from thin singular strands that run parallel to the microvasculature to more complex mesh processes that enwrap the entire vessel lumen. Pericytes residing more proximal to the precapillary arteriole, which are reported to be involved in blood flow control, ${ }^{5}$ exhibit more complex processes that may be essential to their contractile function (Figs. 7 and 10). While many of the morphologies we observed here have been previously described, ${ }^{28,31}$ to our knowledge, this is the first characterization of pericyte branch patterns and process lengths at the level of single cells (Fig. 6).

In a second mouse line driven constitutively by the PDGFR $\beta$ promoter, we find that nearly all pericytes are labeled such that their processes cover the entire capillary bed. Their stereotyped ovoid cell bodies are easily detected in optically cleared ex vivo specimens, allowing for large-scale quantification of pericyte distribution and topological localization (Fig. 9). Our studies suggest that the combined use of NG2-CreER ${ }^{\mathrm{TM}}$ and
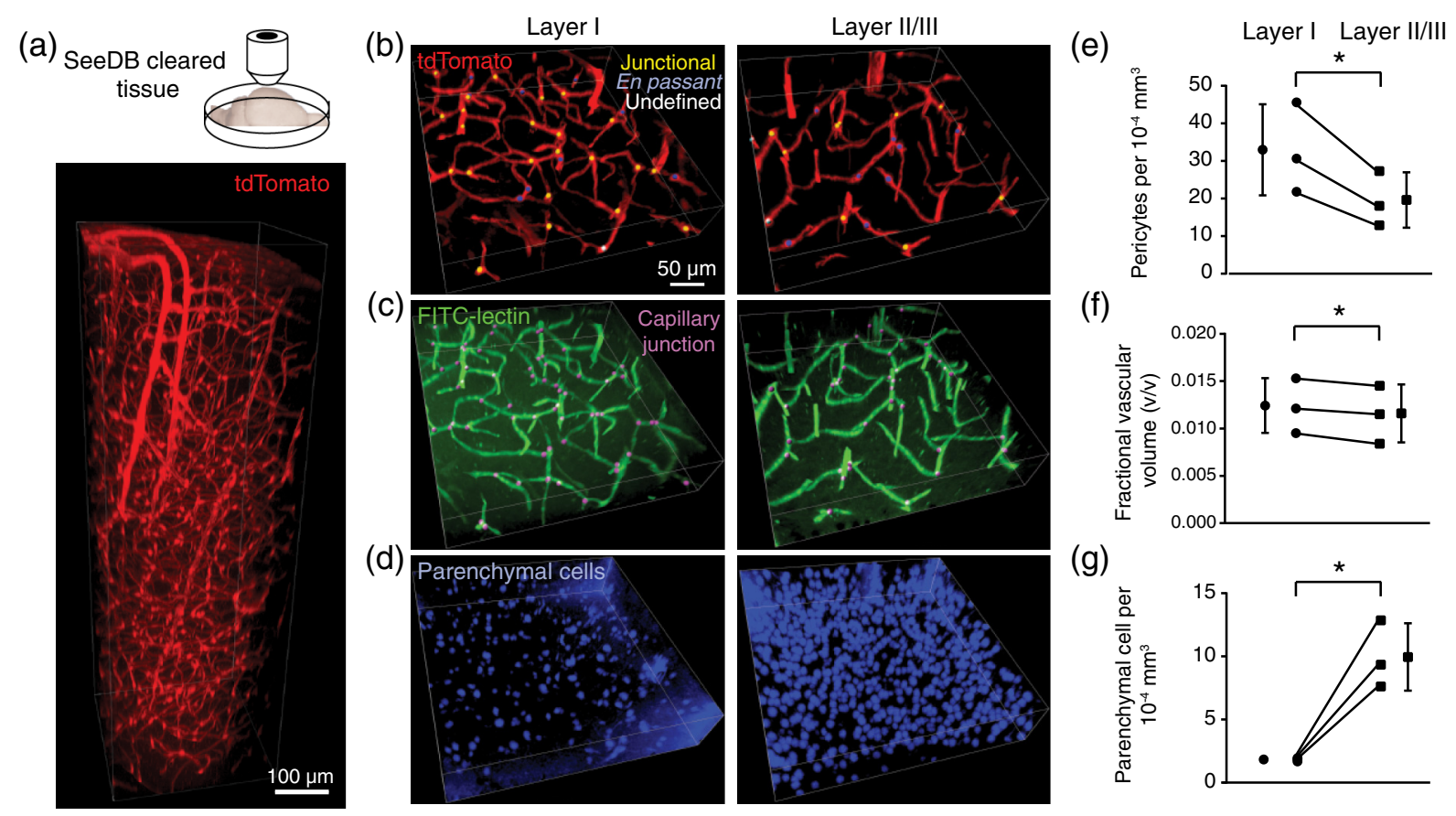

(c)
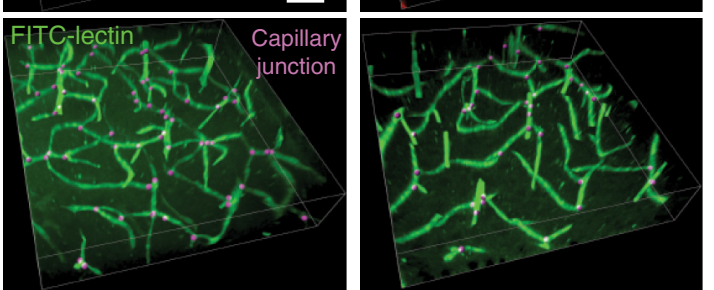

(f)

(d)
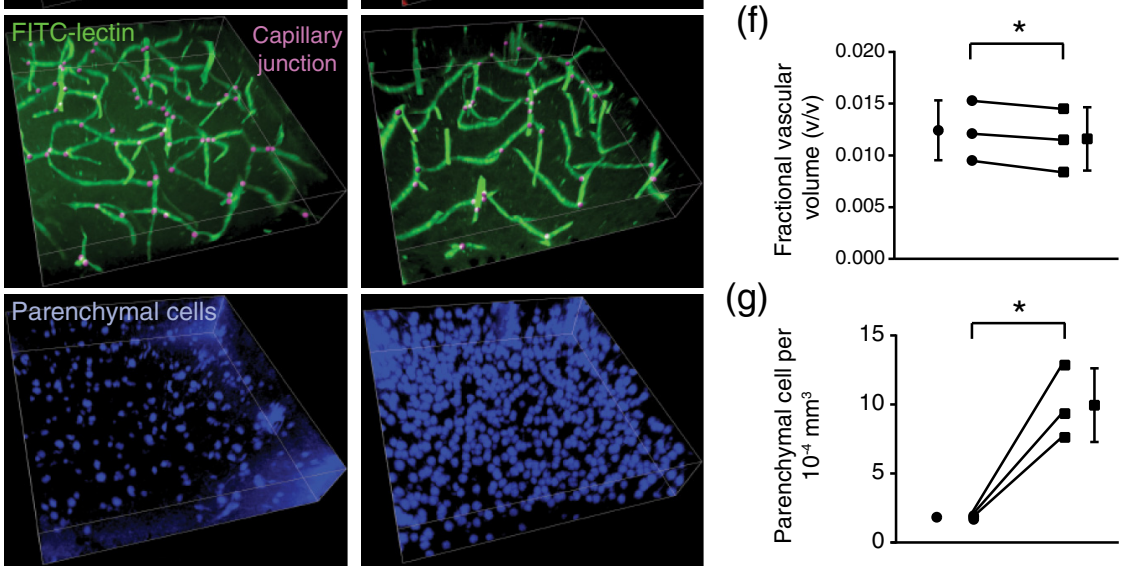

(g)

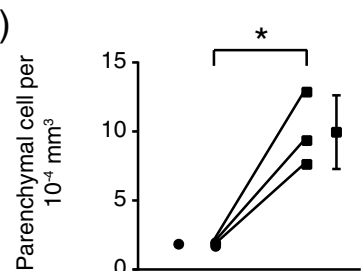

Fig. 9 Large-scale imaging of vascular mural cells using optical tissue clearing and two-photon imaging of PDGFR $\beta$-tdTomato tissues: (a) A volume of cortical tissue spanning 300 by $300 \mu \mathrm{m}^{2}$ in the lateral plane and $1000 \mu \mathrm{m}$ in depth below the pia, imaged ex vivo after tissue clearing with SeeDB. Detailed characterizations of (b) pericyte number and location, (c) vascular volume, and (d) parenchymal cell density in layers I and II/III were collected from these larger datasets. (e)-(g) Statistical comparison of measured parameters between layers I and II/III. The reported values for $(\mathrm{e})$ and $(\mathrm{g})$ are cell numbers in the approximate tissue volumes measured, i.e., $0.0001 \mathrm{~mm}^{3}$. 

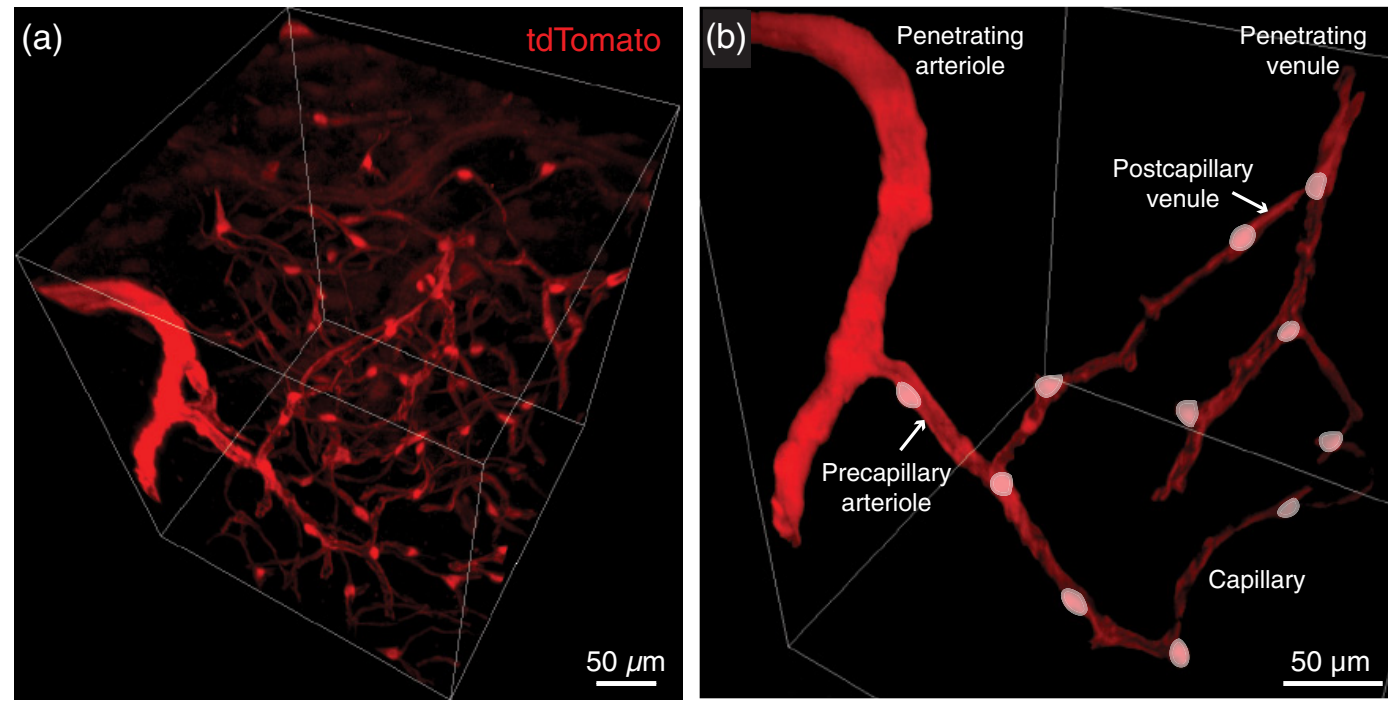

(c) Arterial Smooth muscle-

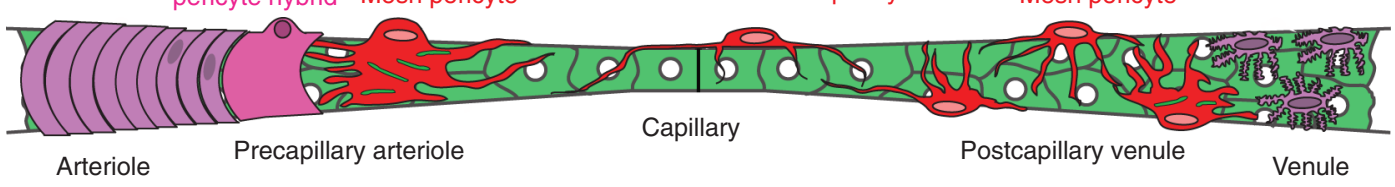

Fig. 10 Summary of mural cell organization in the cerebrovasculature: (a) A tissue volume from a PDGFR $\beta$-tdTomato mouse imaged after optical clearing. (b) Distribution of pericytes and pericyte-smooth muscle hybrids in an arteriole-capillary-venule loop. The cell bodies are labeled in white. (c) Schematic showing the continuum of mural cell types along the cerebral vasculature. Smooth muscle cells form concentric rings on arterioles. Hybrid smooth muscle-pericyte cells reside on precapillary arterioles and interlock with mesh pericytes at the arteriole-capillary interface, which occurs as penetrating arterioles ramify into the capillary bed. Pericytes in capillary beds typically exhibit long processes that traverse the microvasculature in single strands or pairs that twist in a helical fashion. Mesh pericytes become more prevalent again as capillaries turn into postcapillary venules. Stellate-shaped smooth muscle cells cover the walls of parenchymal venules.

PDGFR $\beta$-Cre driver lines will allow characterization of pericytes in any vascular bed of the brain, which can then inform in vivo structure-function studies and computational approaches to understand cerebral blood flow control. ${ }^{32}$

We provide details on the transition of mural cell types from penetrating arterioles to precapillary arterioles to capillaries (Figs. 7, 8, and 10). In particular, imaging of NG2-tdTomato mice revealed smooth muscle-pericyte hybrid cells that abutted mesh pericytes. These two cell types appeared to interlock rather than overlap at the arteriole-capillary interface [Figs. 7(j) and $7(\mathrm{k})$ ]. The ovoid cell bodies of hybrid cells appear much like those of classic pericytes seen within the capillary bed, and this similarity may complicate the ability to distinguish between these cell types in vivo while using NG2 or PDGFR $\beta$ promoterdriven mouse lines. The disparate expression of $\alpha$-SMA between hybrid cells and mesh pericytes suggests distinct functions in blood flow modulation that should be examined in more detail. Further studies are also required to understand the relationship between vascular topology and the distribution of arteriole-capillary interfaces, as this bears relevance to the locus of blood flow control at the level of capillaries. In addition, approaches to genetically separate smooth muscle cells from the pericytes population will be necessary to better study the features of classic pericytes in isolation. ${ }^{17}$

The extent to which the pericytes cover the endothelium has been used as a method to detect pericyte loss or degeneration during age-related blood-brain barrier breakdown. ${ }^{2,6}$ This highlights the need for new methods to characterize the plasticity of pericyte processes, which cover the vast majority of the cerebral vasculature under normal conditions. ${ }^{33}$ A potential caveat, however, is that immunostaining reports protein expression and localization rather than the true structure of the cell, and protein content can be altered in diseased states. ${ }^{34}$ In contrast, fluorescent proteins driven by constitutive promoters that completely fill the cytoplasmic space, in this case tdTomato, will provide a more accurate measure of pericyte structure. The contiguous labeling provided by the PDGFR $\beta$-tdTomato line may be useful in assessing vascular coverage ${ }^{35}$ or pericyte numbers by counting cell bodies [Fig. 9(b)]. Remodeling of fine processes as opposed to overt pericyte loss may also alter vascular coverage. Such subtle changes can escape detection with immunostains or less brightly labeled mouse lines. ${ }^{36}$ NG2-tdTomato mice may be useful in this regard, since specific morphological changes can be clearly visualized and quantified at the level of single pericytes.

Optical tissue clearing and imaging techniques are rapidly advancing, enabling researchers to extract detailed cellular and structural information from large tissue volumes. ${ }^{37}$ Here, we demonstrated the utility of the PDGFR $\beta$-tdTomato mice in optical clearing studies, showing the feasibility of quantifying pericyte distribution within the three-dimensional cerebrovascular network. Our initial data suggest the pericyte number 
is inversely related to parenchymal cell density in upper cortical layers. The data may imply that the layer-specific reductions in the pericyte number limit flow resistance where the metabolic demand is high. Alternatively, a high pericyte number in layer I may serve to buffer high flow pressures from pial surface arterioles. The findings presented here, however, are based on quantifications in a restricted area of cortex, warranting future studies to map pericyte distribution through multiple cortical layers and varying brain regions.

\section{Methods}

\subsection{Animals and Surgery}

TdTomato reporter mice [Jackson Labs; stock no. 007914 (Ai14) $]^{16}$ were bred with two different Cre driver lines: NG2CreER $^{\mathrm{TM}}$ mice $^{20}$ (Jackson Labs; stock no. 008538) and PDGFR $\beta$-Cre mice. ${ }^{19}$ The Cre driver lines were male and the reporters were female in our breeding scheme. Mice were maintained in standard cages on a 12-h light-dark cycle. Male and female mice of 2 to 6 months of age were used. Pericyte density was not expected to change between 1 and 16 months of age in normal mice. ${ }^{6}$ Bigenic PDGFR $\beta$-tdTomato mice were identified by observing tdTomato fluorescence in a tail sample using an epifluorescence microscope (Olympus SZX16). The tail was fileted in order to observe blood vessels on the inner surface of the skin. Bigenic NG2-tdTomato mice were identified by genotyping (Transnetyx). TdTomato expression was then induced in successfully crossed mice by intraperitoneal injection of tamoxifen at a dose of $100 \mathrm{mg} / \mathrm{kg}$ dissolved in corn oil:ethanol (9:1) every $24 \mathrm{~h}$ for 5 consecutive days. NG2-tdTomato mice were then imaged or sacrificed for histology within 2 months after the final tamoxifen injection.

Prior to two-photon imaging, skull-removed cranial windows were generated to obtain optical access to the sensorimotor cortex. ${ }^{38}$ Anesthesia was induced with isoflurane (Patterson Veterinary) at $4 \%$ mean alveolar concentration (MAC) in $100 \%$ oxygen and maintained at $1 \%$ to $2 \%$ MAC during surgery. Body temperature was maintained at $37^{\circ} \mathrm{C}$ with a feedback-regulated heat pad. Imaging was performed within $24 \mathrm{~h}$ of window construction. All animals were administered buprenorphine prior to or immediately following the surgery at a concentration of $0.05 \mathrm{mg} / \mathrm{kg}$ for analgesia. The procedures were approved by the Institutional Animal Care and Use Committee at the Medical University of South Carolina.

\subsection{In Vivo Two-Photon Microscopy}

Two-photon imaging was performed with a Sutter Moveable Objective Microscope (MOM) and a Coherent Ultra II Ti: Sapphire laser source set to 975-nm excitation. Red emission was collected through an ET605/52m bandpass filter (Chroma Technology Corp.). High-resolution imaging was performed using a water immersion $20 \times, 1.0 \mathrm{NA}$ objective lens (Olympus XLUMPLFLN 20XW). Mice were maintained under light isoflurane $(0.5 \%$ to $0.75 \%$ MAC) supplied in moisturized air throughout imaging. Lateral sampling was $0.4 \mu \mathrm{m}$ per pixel and axial sampling was $1 \mu \mathrm{m}$ steps between frames.

\subsection{Immunohistochemistry}

Mice were transcardially perfused with PBS and 4\% paraformaldehyde (PFA) following sacrifice with Euthasol (Patterson Veterinary). Brains were extracted and postfixed overnight at $4^{\circ} \mathrm{C}$. Whole or hemisected brains were mounted and sliced on a Vibratome series 1000 system to yield 50- $\mu \mathrm{m}$ thick coronal sections that were then stored in PBS with $0.02 \%$ azide. When staining with $\alpha$-SMA antibodies (see source below), slices were incubated in PBS with $0.125 \%$ trypsin (Sigma; T4049) for $30 \mathrm{~min}$ at $37^{\circ} \mathrm{C}$ prior to antibody staining followed by 30 min of washing in PBS. This treatment was necessary for antibody access to the vascular media throughout the tissue section and was deemed optimal after testing a range of trypsin concentrations. For immunostaining, slices were first placed into a solution containing primary antibodies diluted into PBS with $10 \%(\mathrm{v} / \mathrm{v})$, goat serum (Vector Labs), $2 \%$ (v/v) Triton X-100 (Sigma), and $0.02 \%(\mathrm{w} / \mathrm{v})$ sodium azide (Sigma). Primary antibodies were used at the following dilutions: 1:500 rat anti-CD13 (Acris Antibodies; AM26636AF-N), 1:100 rabbit anti-PDGFR $\beta$ (Abcam; ab32570), 1:200 rabbit anti-NG2 (Millipore; ab5320), 1:50 rat anti-CD31 (BD Biosciences; 550274), and 1:500 FITC-conjugated mouse anti- $\alpha$-SMA (SigmaAldrich; F3777). After slow nutation overnight at room temperature, the slices were washed in PBS and transferred to secondary antibody, if required, in the same diluent as primary antibodies for $2 \mathrm{~h}$. These secondary antibodies were used at the following dilutions: 1:1000 goat anti-rat Oregon Green 488 (Invitrogen; O-6382) and 1:1000 goat anti-rabbit Alexa 488 (Invitrogen; A31628). In some cases, tissues were additionally incubated for $10 \mathrm{~min}$ with Hoechst (Invitrogen; H3569) diluted to $1 \mu \mathrm{g} / \mathrm{mL}$ in PBS. After washing again in PBS, slices were mounted, dried, and coverslip-sealed with Fluoromount-G (Southern Biotechnology).

Epifluorescence images were acquired using a 10×, $0.3 \mathrm{NA}$ air objective (Olympus UPLFLN10X2) on an Olympus BX53 microscope equipped with a QI Click CCD camera (QImaging). The imaging resolution was $1 \mu \mathrm{m}$ per pixel. Higher resolution confocal images were collected using an Olympus FV10i confocal microscope and a $60 \times 1.2 \mathrm{NA}$ oil immersion objective. Imaging resolution for maximally projected stacks was $0.2 \mu \mathrm{m}$ per pixel in the lateral plane and $1 \mu \mathrm{m}$ per pixel in the axial plane. For reconstructions of pericyte structure (Fig. 6), we used the same lateral resolution, but a higher axial resolution of $0.4 \mu \mathrm{m}$ per pixel.

\subsection{Pericyte Quantification in Histological Slices}

Pericytes were counted in epifluorescence images of tissues stained with CD13. One region of interest was selected for three different cortical slices per animal (Table 1). Cells were independently counted in both tdTomato and CD13 channels. They were categorized as pericytes if they met the following criteria: (1) the cell possessed a small and well-defined ovoid cell body, and (2) there was at least one process emanating from the cell body that was comparable in brightness, but thinner in diameter than the cell body. For both mouse lines, after overlaying both channels, additional cells with pericyte characteristics were discovered, indicating that about $10 \%$ of pericytes were missed in counting with the morphological criteria above. Colabeled cells that were initially missed in one channel were counted toward the total number of pericytes in the other channel. Sensitivity reports the number of tdTomato-positive cells successfully categorized as pericytes. To calculate the sensitivity of labeling, the number of pericytes that colabeled with tdTomato and CD13 was divided by the total number of CD13 pericytes per region of interest. The false positive rate reports the fraction of cells that were mistakenly believed to 
be pericytes based on morphology. To calculate the false positive rate, the number of cells that were counted as pericytes using the tdTomato channel, but were not CD13-positive, was divided by the number of pericytes that were CD13 and tdTomato-positive.

\subsection{Analysis of Pericyte Structure}

Analyses of pericyte structure were performed on volume rendered data from high-resolution confocal stacks using Imaris 7.7 software (Bitplane). Care was taken to only analyze single pericytes that were completely isolated from other fluorescent cells. We created surface renderings of each cell in the tdTomato channel that were smoothed to a level of detail of $\sim 1 \mu \mathrm{m}$, which was half the thickness of thin-strand pericyte processes [Fig. 6(f)]. Smaller, nonspecific structures in the background were removed by thresholding for object size. Pericyte processes were traced in 3-D space using the filament tool to measure their lengths. Individual processes were then isolated to measure surface area and cytoplasmic volume.

\subsection{Tissue Clearing and Imaging}

To obtain high-resolution 3-D images of the cortical vasculature, we utilized the SeeDB optical clearing method. ${ }^{39}$ To label the vasculature, we injected $10 \mu \mathrm{L}$ of $2 \mathrm{mg} / \mathrm{mL}$ FITC-conjugated tomato lectin (Vector labs; FL-1171) retro-orbitally into PDGFR $\beta$-tdTomato mice. Two hours after injection, the mice were perfusion-fixed and the brain extracted and placed in 4\% PFA in PBS overnight. The brain was then hemisected and placed into a series of $\mathrm{D}(-)$ fructose solutions as described in Ke et al. ${ }^{39}$ Once sufficiently cleared, the tissue was submerged in SeeDB solution inside a Petri dish. The surface of the brain was covered with a coverglass and image stacks were obtained at 925-nm excitation with two-photon microscopy. Red and green fluorescence was separated through ET605/52m and ET525/50m bandpass filters, respectively (Chroma Technology Corp.). One millimeter deep image stacks were collected with a lateral resolution of 0.4 to $0.6 \mu \mathrm{m}$ per pixel and an axial resolution of $1 \mu \mathrm{m}$ per frame.

\subsection{Analysis of Pericyte Distribution in Cleared Tissues}

To isolate separate tissue volumes from layers I and II/III, we first counted parenchymal cell bodies in SeeDB image stacks. Parenchymal cell bodies were visible in the green channel due to exclusion of extravasated FITC-lectin. These images were inverted and used to generate a binary mask for cell counts using previously published algorithms. ${ }^{30} \mathrm{~A}$ plot of cell number versus frame was used to map the transition between the cellsparse layer I and cell-dense layer II/III. Since layer I is reported as being $\sim 70 \mu \mathrm{m}$ thick, ${ }^{40}$ we selected the $70 \mu \mathrm{m}$ superficial to the transition of cell density as layer I. An equivalent range of stacks deep to the transition was designated for layer II/III, with the peak cell density set as the center frame.

Quantifications of SeeDB data were performed in Imaris software. Image volumes for layers I and II/III were first median filtered $(3 \times 3 \times 1$ pixels $)$ in both red and green channels to reduce noise. Parenchymal cells were enumerated in intensity-inverted volumes from the green channel, using size and intensity thresholds in Imaris that were made on a case-bycase basis. Our counts are likely to have underestimated the total cells, as FITC-lectin may not be excluded from all cells. The vascular volume was measured in noninverted volumes from the green channel by applying an intensity threshold equal to the mean plus 2 times the standard deviation. Marking of pericyte locations in image volumes from the red channel was performed in 3-D space to best detect vessels that projected axially from a capillary junction, and was performed by an experimenter blind to the cortical layer being analyzed. Only pericytes with the center of their cell body within the image volume were counted toward the total. Pericytes were only designated junctional if the center of the cell body was distributed across the different capillaries converging at that particular junction. All other pericytes were deemed en passant. Pericytes whose locations could not be determined due to their position at the edge of the image volume were labeled as "undefined". There were no instances when a pericyte was too dim to categorize or count.

\subsection{Statistics}

Paired $t$-tests were conducted in Graphpad Prism software when comparing quantification from layers I to II/III [Fig. 9(e)-9(g)]. Three images stacks from one animal were examined. Kolmogorov-Smirnov tests, which do not require assumption of normality, were conducted in MATLAB to test for differences in pericyte structure [Fig. 6(i)].

\section{Acknowledgments}

Our work is generously supported by grants to A.Y.S. from the NINDS (NS085402), the Dana Foundation, the American Heart Association (14GRNT20480366), and South Carolina Clinical and Translational Institute (UL1TR000062). A.Y.S. is also supported by an Institutional Development Award (IDeA) from the NIGMS under grant number P20GM12345. Confocal images were collected at the Cell \& Molecular Imaging Shared Resource of the Hollings Cancer Center at the Medical University of South Carolina (P30 CA138313).

\section{References}

1. C. Rouget, "Memoire sur le developpement la structure et les proprietes physiologiques des capillaires sanguins et lymphatiques," Arch. Physiol. Norm. Path. 5, 603-663 (1873).

2. A. Armulik et al., "Pericytes regulate the blood-brain barrier," Nature 468(7323), 557-561 (2010).

3. R. Daneman et al., "Pericytes are required for blood-brain barrier integrity during embryogenesis," Nature 468(7323), 562-566 (2010).

4. H. Gerhardt and C. Betsholtz, "Endothelial-pericyte interactions in angiogenesis," Cell Tissue Res. 314(1), 15-23 (2003).

5. C. N. Hall et al., "Capillary pericytes regulate cerebral blood flow in health and disease," Nature 508(7494), 55-60 (2014).

6. R. D. Bell et al., "Pericytes control key neurovascular functions and neuronal phenotype in the adult brain and during brain aging," Neuron 68, 409-427 (2010).

7. B. V. Zlokovic, "The blood-brain barrier in health and chronic neurodegenerative disorders," Neuron 57, 178-201 (2008).

8. P. Dore-Duffy, "Pericytes: pluripotent cells of the blood brain barrier," Curr. Pharm. Des. 14, 1581-1593 (2008).

9. M. Yemisci et al., "Pericyte contraction induced by oxidative-nitrative stress impairs capillary reflow despite successful opening of an occluded cerebral artery," Nat. Med. 15, 1031-1037 (2009).

10. S. Lui et al., "The role of pericytes in blood-brain barrier function and stroke," Curr. Pharm. Des. 18, 3653-3662 (2012).

11. A. Armulik, G. Genove, and C. Betsholtz, "Pericytes: developmental, physiological, and pathological perspectives, problems and promises," Dev. Cell 21(2), 193-215 (2011). 
12. H. A. Zariwala et al., "A Cre-dependent GCaMP3 reporter mouse for neuronal imaging in vivo," J. Neurosci. 32(9), 3131-3141 (2012).

13. L. Madisen et al., "A toolbox of Cre-dependent optogenetic transgenic mice for light-induced activation and silencing," Nat. Neurosci. 15(5), 793-802 (2012).

14. F. Fernández-Klett et al., "Pericytes in capillaries are contractile in vivo, but arterioles mediate functional hyperemia in the mouse brain," Proc. Natl. Acad. Sci. U. S. A. 107(51), 22290-22295 (2010).

15. A. Nagy, "Cre recombinase: the universal reagent for genome tailoring," Genesis 26(2), 99-109 (2000).

16. L. Madisen et al., "A robust and high-throughput Cre reporting and characterization system for the whole mouse brain," Nat. Neurosci. 13, 133-140 (2010).

17. D. A. Hartmann et al., "Murine toolbox for imaging the neurovascular unit," Microcirculation 22(3), 168-182 (2015).

18. U. Ozerdem et al., "NG2 proteoglycan is expressed exclusively by mural cells during vascular morphogenesis," Dev. Dyn. 222(2), 218-227 (2001).

19. A. S. Cuttler et al., "Characterization of Pdgfrb-Cre transgenic mice reveals reduction of ROSA26 reporter activity in remodeling arteries," Genesis 49(8), 673-680 (2011).

20. X. Zhu et al., "Age-dependent fate and lineage restriction of single NG2 cells," Development 138(4), 745-753 (2011).

21. N. C. Shaner et al., "Improved monomeric red, orange and yellow fluorescent proteins derived from Discosoma sp. red fluorescent protein," Nat. Biotechnol. 22, 1567-1572 (2004).

22. K. Svoboda and R. Yasuda, "Principles of two-photon excitation microscopy and its applications to neuroscience," Neuron 50, 823839 (2006).

23. J. Kunz et al., "The 140-kDa protein of blood-brain barrier-associated pericytes is identical to aminopeptidase N," J. Neurochem. 62(6), 23752786 (1994).

24. P. Lindahl et al., "Pericyte loss and microaneurysm formation in PDGFB-deficient mice," Science 277, 242-245 (1997).

25. F. Alliot et al., "Pericytes and periendothelial cells of brain parenchyma vessels co-express aminopeptidase $\mathrm{N}$, aminopeptidase A, and nestin," J. Neurosci. Res. 58(3), 367-378 (1999).

26. A. Y. Shih et al., "Two-photon microscopy as a tool to study blood flow and neurovascular coupling in the rodent brain," J. Cereb. Blood Flow Metab. 32(7), 1277-1309 (2012).

27. M. Drobizhev et al., "Two-photon absorption properties of fluorescent proteins," Nat. Methods 8(5), 393-399 (2011).

28. I. Ushiwata and T. Ushiki, "Cytoarchitecture of the smooth muscles and pericytes of rat cerebral blood vessels," J. Neurosurg. 73, 82-90 (1990).

29. I. M. Herman and P. A. D'Amore, "Microvascular pericytes contain muscle and nonmuscle actins," J. Cell Biol. 101, 43-52 (1985).

30. P. S. Tsai et al., "Correlations of neuronal and microvascular densities in murine cortex revealed by direct counting and colocalization of cell nuclei and microvessels," J. Neurosci. 29, 14553-14570 (2009).

31. V. Nehls and D. Drenckhahn, "Heterogeneity of microvascular pericytes for smooth muscle type alpha-actin," J. Cell Biol. 113, 147-154 (1991).
32. P. Blinder and $\mathrm{P}$ et al., "The cortical angiome: an interconnected vascular network with noncolumnar patterns of blood flow," Nat. Neurosci. 16, 889-897 (2013).

33. E. A. Winkler et al., "Blood-spinal cord barrier pericyte reductions contribute to increased capillary permeability," J. Cereb. Blood Flow Metab. 32(10), 1841-1852 (2012).

34. N. Makihara et al., "Involvement of platelet-derived growth factor receptor $\beta$ in fibrosis through extracellular matrix protein production after ischemic stroke," Exp. Neurol. 264, 127-134 (2014).

35. E. A. Winkler, R. D. Bell, and B. Zlokovic, "Central nervous system pericytes in health and disease," Nat. Neurosci. 14(11), 1398-1405 (2011).

36. A. Mishra et al., "Imaging pericytes and capillary diameter in brain slices and isolated retinae," Nat. Protoc. 9(2), 323-326 (2014).

37. P. Osten and T. W. Margrie, "Mapping brain circuitry with a light microscope," Nat. Methods 10(6), 515-523 (2013).

38. R. Mostany and C. Portera-Cailliau, "A craniotomy surgery procedure for chronic brain imaging," J. Vis. Exp. 12, e680 (2008).

39. M. T. Ke, S. Fujimoto, and T. Imai, "SeeDB: a simple and morphologypreserving optical clearing agent for neuronal circuit reconstruction," Nat. Neurosci. 16(8), 1154-1161 (2013).

40. J. DeFelipe, L. Alonso-Nanclares, and J. I. Arellano, "Microstructure of the neocortex: comparative aspects," J. Neurocytol. 31(3-5), 299-316 (2002).

David A. Hartmann is an $\mathrm{MD} / \mathrm{PhD}$ candidate in the laboratory of Andy Shih in the Department of Neuroscience at the Medical University of South Carolina.

Robert G. Underly is a PhD candidate in the laboratory of Andy Shih in the Department of Neuroscience at the Medical University of South Carolina.

Roger I. Grant is a research associate in the laboratory of Andy Shih in the Department of Neuroscience at the Medical University of South Carolina.

Ashley N. Watson is a research associate in the laboratory of Andy Shih in the Department of Neuroscience at the Medical University of South Carolina.

Volkhard Lindner is a senior scientist at Maine Medical Center Research Institute. He is a vascular biologist with numerous publications in the area of vascular repair and remodeling. He developed mouse arterial injury models and discovered the gene Cthrc1.

Andy Y. Shih is an assistant professor in the Department of Neuroscience at the Medical University of South Carolina. His laboratory investigates the mechanisms of microvascular injury and repair during disease using in vivo imaging techniques. 